\title{
Compartment-Resolved Imaging of Activity-Dependent Dynamics of Cortical Blood Volume and Oximetry
}

\author{
Ivo Vanzetta, Rina Hildesheim, and Amiram Grinvald \\ Department of Neurobiology, Weizmann Institute of Science, Rehovot 76100, Israel
}

\begin{abstract}
Optical imaging, positron emission tomography, and functional magnetic resonance imaging (fMRI) all rely on vascular responses to image neuronal activity. Although these imaging techniques are used successfully for functional brain mapping, the detailed spatiotemporal dynamics of hemodynamic events in the various microvascular compartments have remained unknown. Here we used highresolution optical imaging in area 18 of anesthetized cats to selectively explore sensory-evoked cerebral blood-volume (CBV) changes in the various cortical microvascular compartments. To avoid the confounding effects of hematocrit and oximetry changes, we developed and used a new fluorescent blood plasma tracer and combined these measurements with optical imaging of intrinsic signals at a near-isosbestic wavelength for hemoglobin $(565 \mathrm{~nm})$. The vascular response began at the arteriolar level, rapidly spreading toward capillaries and venules. Larger veins lagged behind. Capillaries exhibited clear blood-volume changes. Arterioles and arteries had the largest response, whereas the venous response was smallest. Information about compartment-specific oxygen tension dynamics was obtained in imaging sessions using $605 \mathrm{~nm}$ illumination, a wavelength known to reflect primarily oximetric changes, thus being more directly related to electrical activity than $\mathrm{CBV}$ changes. Those images were radically different: the response began at the parenchyma level, followed only later by the other microvascular compartments. These results have implications for the modeling of fMRI responses (e.g., the balloon model). Furthermore, functional maps obtained by imaging the capillary CBV response were similar but not identical to those obtained using the early oximetric signal, suggesting the presence of different regulatory mechanisms underlying these two hemodynamic processes.
\end{abstract}

Key words: optical imaging; neurovascular coupling; arterioles; CBV; microvasculature; fMRI

\section{Introduction}

More than a century after the seminal work of Roy and Sherrington (1890), neurovascular coupling and its underlying physiological and metabolic processes are still incompletely understood; however, their exploration is accelerating (Pellerin and Magistretti, 1994, 2004; Villringer and Dirnagl, 1995; Magistretti and Pellerin, 1999; Magistretti et al., 1999; Obrig et al., 2002; Caesar et al., 2003a,b; Chatton et al., 2003; Parri and Crunelli, 2003). Part of this growing interest might be explained by the increasing use of hemodynamic-based functional brain mapping techniques such as optical imaging, positron emission tomography, and functional magnetic resonance imaging (fMRI), on one hand, whereas on the other hand, our understanding of the hemodynamic signals with respect to the underlying neuronal activity in terms of input, output, and local processing (Logothetis, 2003 ) is still incomplete. Furthermore, the usefulness of hemodynamic responses for high-resolution functional imaging at the

\footnotetext{
Received July 24, 2004; revised Jan. 7, 2005; accepted Jan. 8, 2005.

This work was supported by grants from the Israeli Science Foundation, the National Institutes of Health, and the Heinemann Foundation. We thank D. Ettner and Drs. I. Lampl and H. Spors for technical assistance and Drs. D. Sharon, F. Chavane, B. Thirion, and 0. Faugeras for critical comments.

Correspondence should be addressed to Ivo Vanzetta, Equipe Dynamique de la Vision et de I'Action, Institut de Neurosciences Cognitives de la Mediterranée, Centre National de la Recherche Scientifique-Unité Mixte de Recherche 6193 (Batiment N'), 31 Chemin Joseph Aiguier, 13402 Marseille, Cedex 20, France. E-mail: ivo.vanzetta@incm.cnrs-mrs.fr.

DOI:10.1523/JNEUROSCI.3032-04.2005

Copyright $\odot 2005$ Society for Neuroscience $\quad$ 0270-6474/05/252233-12\$15.00/0
}

fundamental level of cortical columns remains controversial (Kim et al., 2000; Logothetis, 2000; Duong et al., 2001; Goodyear et al., 2001; Vanzetta et al., 2002, 2004).

To shed light on the spatiotemporal dynamics of the active hemodynamic response in cortex, in the present study we first selectively explored activity-evoked cerebral blood-volume (CBV) changes for the different microvascular compartments by means of high-resolution ( $\sim 7 \mu \mathrm{m} ; 80 \mathrm{~ms})$ optical imaging in anesthetized cat area 18 . The intercompartmental differences in CBV response are small compared with the overall response; therefore, their detailed characterization by reflection recordings at published isosbestic wavelengths may be confounded by small oximetric changes resulting from small deviations of the hemoglobin absorption spectra in vivo with respect to the published in vitro values [in phantom studies, Sheth et al. (2004) found even isosbestic wavelengths to shift by $\sim 2 \mathrm{~nm}$ ] and by eventual hematocrit changes. Therefore, we developed a new long-wavelength (excitation/emission peaks: 640/660 nm) fluorescent bloodplasma tracer that was injected into the blood circulation, where it reached a steady-state concentration such that the fluorescent signal directly reflected CBV changes. We also imaged changes in reflected light at a near-isosbestic wavelength $(\sim 565 \mathrm{~nm})$ for oxyhemoglobin and deoxyhemoglobin ( $\mathrm{HbO} 2$ and Hbr, respectively). Comparison of the obtained CBV time courses with those obtained from the fluorescence measurements allowed us to control for the confounding effects of changes in oximetry and/or 
hematocrit, because these have opposite effects on the fluorescence and the near-isosbestic reflection signals.

Furthermore, oximetric changes were also imaged in the different vascular compartments, using reflection measurements at $605 \mathrm{~nm}$, a wavelength at which Hbr absorbs approximately five times more than $\mathrm{HbO} 2$. This allowed for a comparison of the spatiotemporal dynamics of the active CBV response with those of the early hypo-oxygenation, the "initial dip," which is mainly passive with respect to the vasculature. Indeed, the initial dip at its early phase results mainly from an activity-evoked parenchymal oxygen drop (Ances et al., 2001), correlating with local spiking activity at the spatial scale of cortical columns (Thompson et al., 2003).

Finally, to determine whether different regulatory mechanisms might underlie changes in CBV and oxygen extraction, we compared spatial activation patterns obtained by imaging the early hypo-oxygenation signal and the CBV response at the capillary level.

\section{Materials and Methods}

\section{Preparation}

Experiments were performed on anesthetized (halothane during surgery and sodium pentobarbital during recordings) and paralyzed (succinylcholine chloride) young adult cats $(2-3 \mathrm{~kg})$, with a recording chamber implanted onto the skull above visual area 18 and covered by a glass window. A detailed description of the methodology has been given previously by Bonhoeffer and Grinvald (1993). All procedures were in accordance with National Institutes of Health Guidelines for the Care and Use of Laboratory Animals.

\section{Stimuli}

The visual stimuli used were high-contrast full-field vertical and horizontal drifting square gratings at the optimal spatial and temporal frequency for area 18 , as well as a blank stimulus (dark screen) shown on a computer monitor in front of the cat's eyes. Stimulus onset and optical recording were synchronized to heartbeat and respiration. Each stimulus was averaged at least four times. Stimulus duration was $2 \mathrm{~s}$, whereas the interstimulus interval (dark screen) was $>30 \mathrm{~s}$ to allow for complete relaxation of the hemodynamic response. To verify this, stimuli were presented in the following order: blank, vertical, blank, horizontal, vertical, horizontal. The signal recorded during a given condition after a blank was then compared with the signal recorded during the same condition but after a stimulated condition. No difference was found, and the flat baseline observed confirmed that the hemodynamic response had effectively relaxed during the interstimulus interval.

\section{Optical imaging}

Images were acquired with a commercially available video imaging system (Imager 2001; Optical Imaging, Mountainside, NJ) using a "macroscope" (Ratzlaff and Grinvald, 1991) in epi-illumination mode for the fluorescence measurements and with external illumination 570 or 605 $\mathrm{nm}$ ) through light-guides for the reflection measurements. Two separate tungsten halogen lamps (150 W; Zeiss, Oberkochen, Germany) were used for the two imaging modes. Spatiotemporal resolution was $\sim 7 \mu \mathrm{m}$ and $80 \mathrm{~ms}$. Because the goal was to image vascular responses, the optics were focused on the cortical vasculature, as opposed to common practice during which the focus is positioned $300-600 \mu \mathrm{m}$ below the cortical surface. The measurements during $570 \mathrm{~nm}$ illumination were interlaced with the fluorescence ones with the help of a computerized electromechanical setup after each completion of an entire set of six stimuli. The $605 \mathrm{~nm}$ sessions were performed immediately preceding tracer injection.

\section{Fluorescence measurements using a newly developed blood plasma tracer}

To minimize the contribution of intrinsic chromophores (in particular hemoglobin) to the fluorescence CBV measurements, we synthesized a fluorescent dye with excitation and emission maxima at long wavelengths (640 and $660 \mathrm{~nm}$ ), far outside of the range of high hemoglobin absorption. A cyanine dye (RH1795) was conjugated to a high molecular weight $(70,000)$ dextran, yielding a compound (RH1795-dextran) that, when injected into the bloodstream, did not leak from the cerebral (micro-) vasculature into the parenchyma. The compound was well tolerated, and no change in physiological parameters (expired $\mathrm{CO}_{2}$, heart rate) was noticed after the injections, which were performed every $\sim 2 \mathrm{~h}$ to ensure that, at excitation wavelength $(630 \mathrm{~nm})$, the absorption caused by the dye was large with respect to that caused by hemoglobin. Because the distribution of the fluorescent contrast agent was homogeneous in the blood plasma and could be safely considered stationary over the time of the stimulus-evoked CBV responses, the sensory-evoked fluorescence intensity changes directly reflected changes in CBV. The fluorescence was imaged using the following filter combination: heat-filter (KG1; Schott, Mainz, Germany), excitation at $630 \mathrm{~nm}$ (10 nm bandwidth), dichroic mirror with cutoff at $650 \mathrm{~nm}$ (630DF10 and 650DRLP; Omega Optical, Brattleboro, VT), and long-pass emission with cutoff at $665 \mathrm{~nm}$ (RG665; Schott).

\section{Reflection measurements}

Four different reflection measurements were performed: imaging at a near-isosbestic wavelength $(565 \mathrm{~nm})$ as well as at an oximetric wavelength $(605 \mathrm{~nm})$ and photodiode recordings at an isosbestic wavelength $(570 \mathrm{~nm})($ Sheth et al., 2004) as well as at a fluorescence excitation wavelength $(630 \mathrm{~nm})$.

Imaging. The cortex was illuminated through two light guides after passing through a heat-filter (KG1; Schott) and a 570 or $605 \mathrm{~nm}$ interference filter (570DF10 and 605DF10; Omega Optical). To image at 565 $\mathrm{nm}$ while simultaneously recording reflection changes at the isosbestic wavelength of $570 \mathrm{~nm}$ (Sheth et al., 2004) through a photodiode (see below, Photodiode measurements), we illuminated the cortex with the $570 \mathrm{~nm}$ filter, which had a bandwidth of $10 \mathrm{~nm}$. This finite bandwidth allowed us to take advantage of the spectral properties (non-flat transmission spectrum below $\sim 590 \mathrm{~nm}$ ) of the dichroic mirror used for the fluorescence measurements to obtain the desired $\sim 5 \mathrm{~nm}$ shift of the reflected $570 \mathrm{~nm}$ light to the short side of the spectrum. Unlike the 665 nm cutoff emission filter, the dichroic mirror thus was not removed from the optical path from cortex to video camera for those reflection measurements. With the caveat of changes in hematocrit, the imaged $565 \mathrm{~nm}$ reflection signal was thus mainly sensitive to $\mathrm{CBV}$ changes, with the addition of a small oximetric component introduced by the $\sim 5 \mathrm{~nm}$ shift off the isosbestic point at $\sim 570 \mathrm{~nm}$.

On the other hand, to image oximetric changes, the cortex was illuminated at $605 \mathrm{~nm}$ without the dichroic mirror (and without the $665 \mathrm{~nm}$ emission filter) in the optical path. At $605 \mathrm{~nm}$, changes in [Hbr] are enhanced approximately five times with respect to those of oxyhemoglobin $([\mathrm{HbO} 2])$ (ratio of the extinction coefficients at $605 \mathrm{~nm}: \epsilon_{\mathrm{Hbr}} / \epsilon_{\mathrm{HbO} 2}$ $\sim 5$ ), and thus the signals recorded during illumination at $605 \mathrm{~nm}$ had a strong oximetric component. Because after the contrast agent injection the $605 \mathrm{~nm}$ signal also was dominated by CBV changes caused by the large absorption of the dye at that wavelength (approximately one-half of peak absorption), the $605 \mathrm{~nm}$ imaging session was performed before the injection of the fluorescent dye.

Photodiode measurements. To verify that changes in oximetry and eventually of red blood cell (RBC) density had opposite effects on the fluorescence and reflection signals, we recorded the light backscattered from the imaged area at $630 \mathrm{~nm}$ and at "true" $570 \mathrm{~nm}$ simultaneously with fluorescence and $565 \mathrm{~nm}$ reflection imaging, respectively. For this purpose, we used a photodiode that "saw" the cortex through a third light guide with neither dichroic mirror nor emission filter in the optical path. The area "seen" by the photodiode was matched with that seen by the camera by shining light into the photodiode end of the light guide and adjusting the position of the "cortical" end of the light guide such that the exiting light illuminated an area that coincided with the imaged one.

\section{Wavelength "tuning" of the optical signals}

To control the CBV measurements for oximetric and hematocrit-related artifacts, we imaged blood volume changes in the various microvascular compartments using two different signals that were weakly but oppositely affected by those potential confounds. 
Fluorescence imaging. Despite the dominant absorption attributable to the dye, the large absorption difference between $\mathrm{Hbr}$ and $\mathrm{HbO} 2$ at excitation and emission wavelength [ratio of published extinction coefficients $\left.\epsilon_{\mathrm{Hbr}} / \epsilon_{\mathrm{HbO} 2}(630 \mathrm{~nm}) \sim 6\right]$ introduces a weak dependence of the fluorescent signal on oximetry, in addition to the dominant sensitivity to CBV changes.

Reflection imaging. Imaging at $565 \mathrm{~nm}$ yielded a signal reflecting mainly CBV changes, with a small oximetric component in favor of $[\mathrm{Hbr}]\left[\epsilon_{\mathrm{Hbr}} / \epsilon_{\mathrm{HbO} 2}(565 \mathrm{~nm}) \sim 1.2\right]$. The two blood volume measurements were obviously affected by oximetry- and hematocrit-related effects. For instance, an [Hbr]/[HbO2] ratio increase causes absorption to increase at all wavelengths involved in either fluorescence or reflection measurements; however, increased absorption counteracts the activityevoked fluorescence signal, which is positive, whereas it contributes to the reflection signal at $565 \mathrm{~nm}$, which is negative. The effects on the amplitude of the recorded sensory-evoked CBV-dependent signals is thus opposite in the two imaging modalities. For the same reasons, changes in hematocrit (RBC density) also have opposite effects on the two signals. Furthermore, in the case of fluorescence, an increase in RBC density causes an additional decrease in the recorded signal because of the reduced volume available for fluorescent tracer molecules in the blood vessel. The same reasoning holds true for the effects of static or dynamic differences in oximetry or hematocrit among the various microvascular compartments.

\section{Effects of light scattering}

Because of the strong light scattering of the cortical tissue, the optical response recorded from a given microvascular compartment is somewhat contaminated by contributions from all of the others. Indeed, Orbach (1988) and Orbach et al. (1985) estimated the "smearing" of a local optical signal to be of $\sim 200 \mu \mathrm{m}$. This explains why in the present measurements the recorded response was found to begin approximately simultaneously in all microvascular compartments; however, we could rule out the possibility that the obtained intercompartmental differences in the recorded CBV dynamics were confounded by those nonlocality effects. Indeed, although light scattering itself is nearly constant in the wavelength range used, its effects on the recorded signals are tightly linked to absorption (Kohl et al., 2000), which was estimated to vary by at least one order of magnitude between the wavelengths used in the reflection and fluorescence measurements. Nonlocality effects are thus very different at the two performed measurements and so is the cross-talk among compartments. On the other hand, the differences obtained among the time courses of the various compartments were similar during reflection and fluorescence. This shows that, overall, the artifacts attributable to intercompartmental cross-talk were small compared with the effective differences in CBV response existing among different microvascular compartments.

Activity-evoked light-scattering changes have been reported in bloodless in vitro preparations (Hill and Keynes, 1949; Lipton, 1973; Grinvald et al., 1982; MacVicar and Hochman, 1991) as well as in vivo (Rector et al., 2001). Again, however, their effects are opposite in reflection and fluorescence imaging, and thus these potential confounds on the CBV dynamics obtained here could be ruled out. With respect to the measurements performed at $605 \mathrm{~nm}$, it has been suggested that the contribution of those activity-evoked light-scattering changes to the intrinsic optical signals would become relevant mainly at long wavelengths (Frostig et al., 1990; Mc Loughlin and Blasdel, 1998) because of the low absorption of hemoglobin above $\sim 600 \mathrm{~nm}$. Still, the amplitude of the light-scattering signals recorded in vivo by Rector et al. (2001) even at $780 \mathrm{~nm}$ was only $\sim 5 \times 10^{-5}$ (i.e., nearly two orders of magnitude smaller than the $605 \mathrm{~nm}$ signal recorded in our study). In light of these small amplitudes, the need encountered by Malonek and Grinvald (1996) to invoke light-scattering changes to explain their imaging spectroscopy data (range, $500-650 \mathrm{~nm}$ ) is likely to be the result of simplifications in the spectroscopic decomposition that they used. Indeed, more recent studies using an improved spectroscopic model concluded that the intrinsic signal can be explained by the effects of changes in [Hbr] and [HbO2] alone (Kohl et al., 2000; Lindauer et al., 2001). In these studies, the wavelength dependence of the optical-path length in tissue was taken into account; indeed, with their model, the fit of the spectroscopic data (up to $805 \mathrm{~nm}$ ) was more accurate. In light of those results, we conclude that the contribution of lightscattering changes to the $605 \mathrm{~nm}$ signal was negligible with respect to its hemodynamic component.

\section{Data analysis}

For data analysis, vascular elements belonging to the different compartments were manually chosen by selecting subregions of the image. Noncapillary vascular elements were taken as subregions of the image corresponding to blood vessels or blood vessel branches. To study the activityevoked capillary hemodynamic response, we chose subregions devoid of visible blood vessels. From the neuronal point of view, however, each of the used oriented stimuli activated only a subset of the imaged area. This raised the question of whether to exclude vessel elements located outside of the activated orientation columns from the sample chosen to represent a given microvascular compartment. Indeed, recording also from outside of the activated columns potentially falsifies the comparison among the responses of the different vessel types as a result of a bias in favor of the vascular compartments characterized by the less local responses. On the other hand, restricting the vessel samples to the activated columns has the drawback of reducing the signal-to-noise (SNR) level of the obtained time courses. To evaluate the best strategy to adopt, we calculated the time courses of all compartments twice, first including and then excluding from our samples the vessels lying outside of the cortical columns activated by a given stimulus. Those columns were identified with the functional patches obtained during differential mapping at $605 \mathrm{~nm}$, which are known to identify regions of increased neuronal activity (Bonhoeffer and Grinvald, 1991; Shmuel and Grinvald, 1996; Wang et al., 1996; Weliky et al., 1996; Bosking et al., 1997). At 605 nm, the relationships between the various $605 \mathrm{~nm}$ time courses were slightly different, depending on which calculation was used (see supplemental Fig. 1, available at www.jneurosci.org as supplemental material). The $605 \mathrm{~nm}$ time courses presented here were thus calculated after restricting the samples representative for the different compartments to the active columns. On the other hand, in the CBV measurements, the amplitude and timing relationships among the various time courses were the same, independent of the adopted strategy (see supplemental Fig. 1, available at www. jneurosci.org as supplemental material), consistently with CBV responses being only weakly local. To maximize the SNR, the CBV time courses shown here were thus calculated by selecting the various vessel types from the entire imaged area.

The variability over time ( $\sim 30 \mathrm{~min})$ of the microvascular responses, presumably attributable to the physiological state of the animal, is comparable with or even larger than the differences between microvascular compartments, calling for particular care in analyzing the data. This variability, however, affects the hemodynamic response at the overall level. Thus, the intercompartmental differences were recovered by performing statistical analysis (1) on the compartment-specific responses of many samples of each vascular compartment, each sample being averaged over the same set of trials, and, in addition, (2) after subtracting, for each trial, the response averaged over all compartments from the time courses of each specific compartment, thus drastically reducing the intertrial variability.

(1) Statistical analysis (yielding the error bars appearing in Figs. 4, 5, and 8) was performed on samples of the microvascular compartments chosen from the same averaged dataset. In particular, for one averaged (32-64 repetitions) series of time frames, $\sim 20$ subregions of the image were selected for each one of the five compartments (arteries, arterioles, parenchyma, venules, and veins), thus yielding 20 "arterial" time courses, 20 "arteriolar" ones, and so forth. The statistical analysis was then performed on this dataset, consisting of $\sim 20$ samples for each microvascular compartments (i.e., altogether $\sim 20 \times 5=100$ time courses). The results were independent of the particular set of repetitions averaged, and the same results as those reported here were found in five experiments on three cats. To obtain the statistical distribution of timings regardless of the amplitude of each curve, the [0-1] normalized time courses in Figures 4,5 , and 8 were calculated by scaling each one of those $\sim 100$ curves to $[0,1]$ before computing the mean and the SD.

(2) For both the CBV and the $605 \mathrm{~nm}$ measurements, a large dataset 
(128 and 32 repetitions, respectively) was divided into subsets (16 and 8 , respectively) by averaging together groups of 8 and 4 repetitions, respectively. For each subset, the specific response of each of the five vascular compartments was obtained by averaging over $\sim 20$ samples [see (1) above], yielding five curves for each data subset. Then the average over those five curves was taken, yielding a "mean vascular response" for each data subset. Finally, this mean vascular response was subtracted from the former curves, for each of the 16 or 8 data subsets, respectively, for the case of the CBV and the $605 \mathrm{~nm}$ measurements, and statistical analysis was performed on the results. This method yielded the results shown in Figure 9. Again, for the normalized display, each one of the $16 \times 5$ or $8 \times 5$ curves, respectively, was first rescaled, and then the mean and the SD were computed.

\section{Results \\ Selective identification of the various microvascular compartments by spectral image analysis}

To selectively study the hemodynamics of different cortical microvascular compartments, we identified veins, venules, capillaries, arterioles, and arteries. This was done first by visual inspection (morphology, pulsation, color of vessels, etc.) through a microscope and a camera (Fig. $1 A$ ). This classification was then integrated with oximetric information obtained from an "oximetric image" of the vasculature (Fig. $1 B$ ): two images of the cortical surface were taken at 540 and $560 \mathrm{~nm}$ illumination (peaks of $\mathrm{HbO} 2$ and Hbr absorption, respectively). One image was then divided by the other and the grayscale of the resulting oximetric image was shifted, such that the parenchyma, which contains mainly capillaries, appeared to be in the middle of it (gray). With this procedure, the various vessel types in the image thus were shifted automatically to the bright or dark half of the grayscale according to their different degrees of oxygenation, allowing for further identification of veins and venules (bright) as well as of arteries and arterioles (dark). The direct observation of surface capillaries was only rarely possible at this magnification. Because of the known morphology of the microcirculation (most of the capillary network is buried inside the cortex) (Patel, 1983; Harrison et al., 2002), it is clear, however, that signals originating from parenchymal regions without visible blood vessels (Fig. $1 A, C, D$ ) contained mostly capillary contributions.

\footnotetext{
Activity-dependent oximetric and red blood cell density changes The long-wavelength fluorescent blood-plasma tracer was injected into the blood circulation where it reached a steady-state concentration, and thus changes in fluorescence were directly related to changes in CBV. The influence of intrinsic absorption changes on the fluorescence signal was kept small by ensuring that the contribution of the dye to the total optical density of the blood was several times larger than that attributable to hemoglobin, as shown by Figure 1, $E$ and $F$. At excitation wavelength, the ratio between the baseline absorption attributable to the dye and that attributable to hemoglobin was between 2 and 6 or even more, increasing with the oxygen saturation in the particular vessel. Such high absorption could be achieved using relatively
}
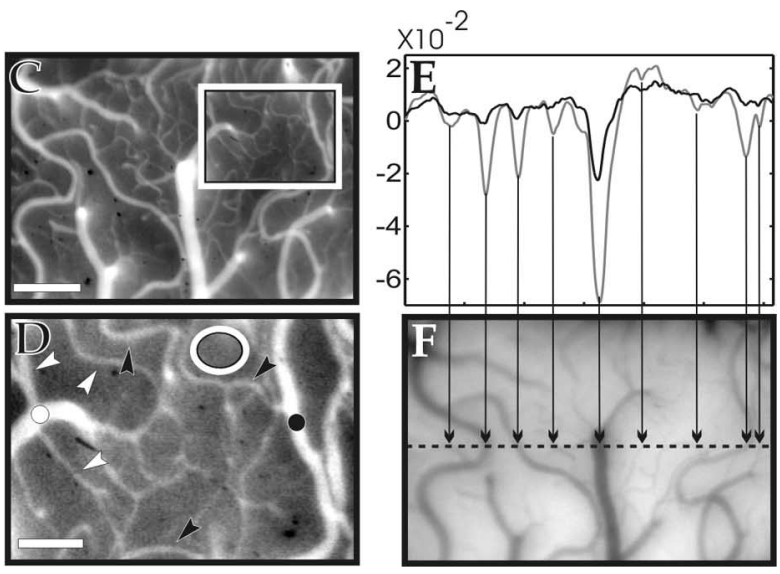

Figure 1. Visualization and identification of the different microvascular compartments. $A$, Image of the cortical surface taken blood vessels. $\boldsymbol{B}$, Ratio of an image taken at $540 \mathrm{~nm}$ and one at $560 \mathrm{~nm}$. This oximetric image aided the identification of the mosty capla whe arowheads; the artery is indicated by a black dot; the vein is indicated by a white dot, parenchyma (i.e. (he dinection; gray, after the dye had reached a stationary distribution in the bloodstream. The different amplitudes of the $\mathrm{nm}$; the dashed line shows the position of the intensity profile in $\boldsymbol{E}$. The arrows relate the dips in the intensity profile in $\boldsymbol{E}$ to locations where the section crosses blood vessels. Scale bars: $\boldsymbol{C}, 0.5 \mathrm{~mm} ; \boldsymbol{D}, 0.2 \mathrm{~mm}$.

low dye concentrations, which did not affect the physiology of the preparation because, unlike in previous studies using Texas Red (Frostig et al., 1990), the excitation and emission peaks of the probe were far from the range of high hemoglobin absorption.

In previous reports (Grinvald et al., 1986; Frostig et al., 1990; Malonek et al., 1997; Vanzetta and Grinvald, 1999, 2001; Erinjeri and Woolsey, 2002; Nemoto et al., 2004), the activity-dependent CBV changes have been studied simply by imaging reflection changes at published isosbestic wavelengths. Because of the large amplitude of the overall CBV response investigated in those studies, it was possible to ignore several possible artifacts affecting the measurements, notably the effects of oximetric contributions attributable to small deviations of the hemoglobin absorption spectra in vivo with respect to the published in vitro values (but see Sheth et al., 2004), RBC density (hematocrit) changes and static and dynamic intercompartmental inhomogeneities, nonideal illumination filters of finite width, and possibly light-scattering changes.

Intercompartmental differences, however, are at least one order of magnitude smaller than the overall response. Thus to study them systematically and reliably, it is necessary to control for the above-mentioned potential confounds.

We therefore controlled the fluorescence measurements for the small oximetric component that still remained, as well as for hematocrit-related effects. This was achieved by comparing the results obtained using fluorescence with those obtained from reflection measurements performed at a near-isosbestic wavelength $(565 \mathrm{~nm})$. The same timing relationships and, to a certain extent, the same amplitude relationships were obtained in the two imaging modes, although the two measurements were affected oppositely (see Materials and Methods) by the above confounds, thus validating our results.

The oximetric dependences described above were verified by comparing the fluorescence and reflection (effective $565 \mathrm{~nm}$ ) 


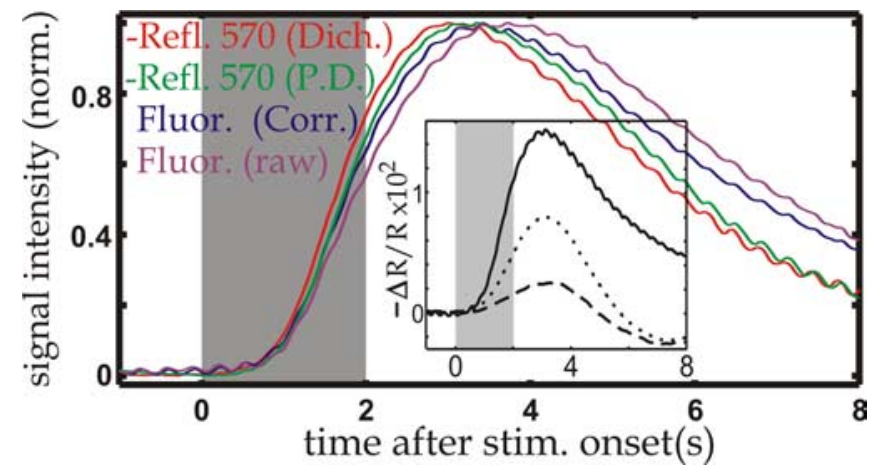

Figure 2. Comparison of the signals recorded with different modalities. Time courses represent the signal averaged over the entire imaged area and were normalized to $[0,1]$; red, $570 \mathrm{~nm}$ imaged through a dichroic mirror (Dich.), $565 \mathrm{~nm}$ reflection (Refl.) signal; green, $570 \mathrm{~nm}$ reflection signal as recorded by the photodiode (P.D.) simultaneously with $565 \mathrm{~nm}$ imaging; magenta, fluorescence amplitude time course; blue, oximetry-corrected fluorescence time course (Corr.): the raw fluorescence was divided by the reflection signal at excitation wavelength (630 $\mathrm{nm})$, recorded by the photodiode during fluorescence imaging. Inset, Reflection (R) signal at $630 \mathrm{~nm}$, just after injection of the dye (solid), $4 \mathrm{~h}$ afterward (dotted line), and $14 \mathrm{~h}$ after injection (dashed line). The large difference between the solid curve and the dashed curve shows that for the data obtained $<2$ h after injection (Figs. 3-6,9,10), the absorption caused by the dye was much larger than that caused by hemoglobin. Here and in all subsequent figures, the grayshaded area marks the time during which the stimulus was presented.

form the correction in single vessels; thus, throughout the results, all compartment-resolved fluorescence time courses are uncorrected. Although this division corrected for the effects of oximetric changes at excitation wavelength, the obtained time course (Fig. 2, blue) was still delayed with respect to that obtained during reflection at $570 \mathrm{~nm}$ (Fig. 2, green), partially because of oximetric changes at emission wavelength, which were not taken into account. Those remaining oximetric changes, however, cannot account for the increasing difference between the two time courses (blue vs green) after $\sim 5 \mathrm{~s}$. Indeed, the difference between the 565 and $570 \mathrm{~nm}$ reflection time courses (Fig. 2, red vs green) was nearly zero between $\sim 7$ and $8 \mathrm{~s}$, indicating that at those times oximetric effects were negligible. On the other hand, changes in hematocrit also contribute to the observed differences between the corrected fluorescence and the $570 \mathrm{~nm}$ reflection time courses. For instance, the contribution of a decrease in RBC density to those discrepancies is even threefold; absorption decreases both at $570 \mathrm{~nm}$ and at fluorescence emission, and furthermore, additional volume becomes available for the fluorescent tracer. None of those effects was taken into account in our correction procedure. The difference between the two time courses thus should be particularly sensitive to changes in RBC density. Indeed, with the caveat of the different penetration depth of 570 versus 630-670 nm light, which might also have contributed to the discrepancies of the observed time courses, the present results are consistent with a decrease in hematocrit, reaching its minimum slightly after the blood flow attains its peak, as reported by Kleinfeld et al. (1998). Because local hematocrit and oximetric changes clearly differ from one vascular compartment to the other, they potentially affect the intercompartmental differences in the recorded blood-volume signals and thus must be controlled for. This underscores once more the importance of the present approach, which combines two measurements in which those confounds have opposite effects.

\section{Activity-dependent blood volume changes in different vascular compartments}

Figure 3 shows the onset of the CBV response, visualized by dividing a stimulated

changes imaged with the camera with the reflection changes recorded simultaneously during $570 \mathrm{~nm}$ illumination by a photodiode (effective $570 \mathrm{~nm}$ ). We found that the fluorescence time course (Fig. 2, magenta) was delayed with respect to the $570 \mathrm{~nm}$ signal recorded by the photodiode (green). Consistently, the normalized time course obtained from the camera recordings during reflection (red) was slightly faster than those recorded by the photodiode (green). These results are expected from underlying oximetric changes similar to those that have been found in several previous studies (Malonek and Grinvald, 1996; Mayhew et al., 1999, 2001; Vanzetta and Grinvald, 1999; Lindauer et al. 2001; Sheth et al., 2004).

To check for the presence of effects attributable to changes in $\mathrm{RBC}$ density, we corrected the overall fluorescence time course for absorption changes at excitation wavelength, dividing it by the $630 \mathrm{~nm}$ reflection time course recorded simultaneously through a photodiode. Note that because the photodiode recorded from the entire imaged area, it was not possible to per- by a blank condition and displayed at high time resolution $(80 \mathrm{~ms}$ per frame). By comparing the emerging vascular structures (bright) in the two panels, it is apparent that as a result of the stimulus in both fluorescence (Fig. $3 A$ ) and near-isosbestic reflection (Fig. $3 B$ ) mode, small arterioles were the first vascular elements to become visible, i.e., to dilate. The active vascular response thus started at the arteriolar level.

Figure 4 shows the time courses of the response for all vascular components, as identified using the procedure illustrated in Figure $1 A-D$, at a time resolution of $80 \mathrm{~ms}$, from $0.5 \mathrm{~s}$ before until $5 \mathrm{~s}$ after stimulus onset.

These curves show that there were clear differences in response among the various vascular compartments. Although arterioles led the response, the vasodilatation propagated quickly to the other vascular elements (Fig. $4 E, F$ ). After $\sim 2-3 \mathrm{~s}$, the signal originating from the arteries caught up with that originating from the arterioles (Fig. 4A, B), and after a peak at 3-4 s, the responses decayed with timing relationships that were opposite those ob- 
served during the rising phase. The amplitudes of the arterial-arteriolar response were largest, followed by venules and parenchyma, whereas the smallest responses were detected in veins. The venous response was also slowest, peaking $0.5-1 \mathrm{~s}$ after the arterioles. The timing of venules and parenchyma was found to be intermediate between that of arterioles and veins. The $565 \mathrm{~nm}$ reflection data displayed several differences as compared with the fluorescence recordings. In general, the reflection signals reached their peak $\sim 500$ $\mathrm{ms}$ earlier than the fluorescent ones (Fig. $4 C, D$ ), with the exception of the venous signal, which in fluorescence mode peaked $>1$ s later than in the reflection recordings. Also, the temporal delay between arterioles and venules was reduced. Finally, the amplitude ratios among the various responses were somewhat different in the $565 \mathrm{~nm}$ reflection and the fluorescence recordings. Given the opposite sensitivity of the two measurements to oximetry- and hematocrit-related effects, those observed discrepancies are most likely caused by differences in oxygenation and RBC density existing among the various vessel types as well as by their activitydependent changes.

To better visualize the intercompartmental differences at early times, in Figure 5 we redrew the data shown in Figure 4, relative to the overall CBV time course. Two types of displays are used, with and without normalization of the various peaks. Without normalization (Fig. $5 A, B, E, F$ ) the display emphasizes the amplitude relationships among the different vascular compartments. Starting with the beginning of the response, vessels such as arterioles and arteries, endowed with active control mechanisms, dilated the most, whereas the passively inflating vessels, and veins in particular, dilated the least.

The normalized display (Fig. $5 C, D$ ), obtained by dividing the time course of each individual vessel by its peak amplitude, emphasizes the characteristics of the response dynamics typical of each microvascular compartment.

Here the arterial time course initially lagged behind the response of capillaries and venules (Fig. 5C,D). A possible explanation for such a relationship is increased arterial outflow through the arterioles, which are the first to dilate, causing a decrease in intra-arterial pressure counteracting active arterial dilatation. The opposite is true for the capillary and venular compartments, in which the increased inflow through the arterioles raises the intravascular pressure.

Subtracting the mean time course from the response also allows for a better display of the spatial patterns of the fluorescence and the reflected light at the various stages of the response, in particular for times $>1 \mathrm{~s}$; without the subtraction, the overall signal change takes up most of the dynamic range of the images. In Figure 6 we therefore centered the grayscale of each image around its mean value of fluorescence (average over all pixels for each point in time), or reflectance in the case of the $565 \mathrm{~nm}$ measurements, thus optimally matching the dynamic range of the grayscale to the spatial patterns of the images. To increase the $\mathrm{SNR}$, the frames, originally acquired at $80 \mathrm{~ms}$ per frame, were binned to $400 \mathrm{~ms}$ per frame.

The different clipping ranges, used at different times, show
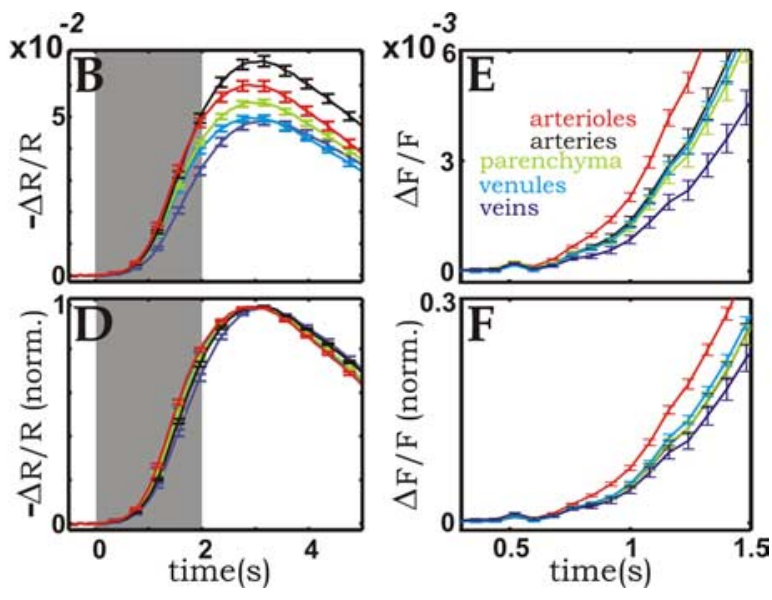

Figure 4. Compartment-resolved time courses of the blood-volume changes in the cortical microvasculature. $\boldsymbol{A}$, Fluorescence vascular compartment chosen from the images. To enhance timing relationships, $\boldsymbol{C}$ and $\boldsymbol{D}$ show the same data as $\boldsymbol{A}$ and $\boldsymbol{B}$ res shown in $\boldsymbol{A}$ and $\boldsymbol{C}$. Here and in all other figures, the error bars represent 1 SD of the mean. Black, Arteries; red, arterioles; light green, parenchyma; cyan, venules; blue, veins.

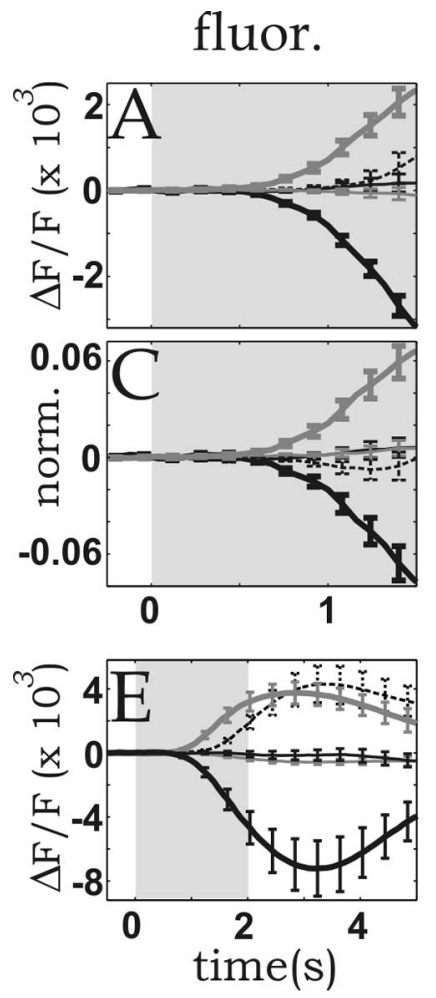

near-isosb.refl.
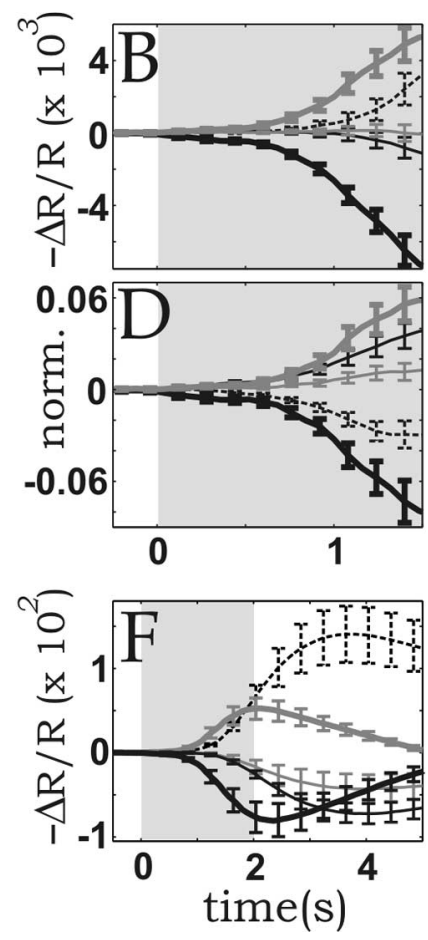

Figure 5. Differences between the compartment-resolved CBV time courses measured by fluorescence (fluor.) and near-isobestic reflection (near-isosb.refl.) in the cortical microvasculature. To emphasize intercompartmental differences, the curves were plotted after subtracting from each of them, for each time point, the mean vascular response (obtained by averaging over all compartments). $\boldsymbol{A}$, Fluorescence amplitude time course of the different microvascular compartments. $\boldsymbol{B}$, Time course of the changes in reflection at $565 \mathrm{~nm}$. $\boldsymbol{C}$ and $\boldsymbol{D}$ are the same as $\boldsymbol{A}$ and $\boldsymbol{B}$, respectively, but baseline-to-peak responses were normalized (norm.) before averaging. $\boldsymbol{E}$, $\boldsymbol{F}$, Non-normalized fluorescence and $565 \mathrm{~nm}$ reflection time courses, respectively, averaged over five experiments performed on three cats. Error bars represent 1 SD of the mean. Dashed lines, Arteries; thick gray lines, arterioles; thin gray lines, parenchyma; thin black lines, venules; thick black lines, veins. 

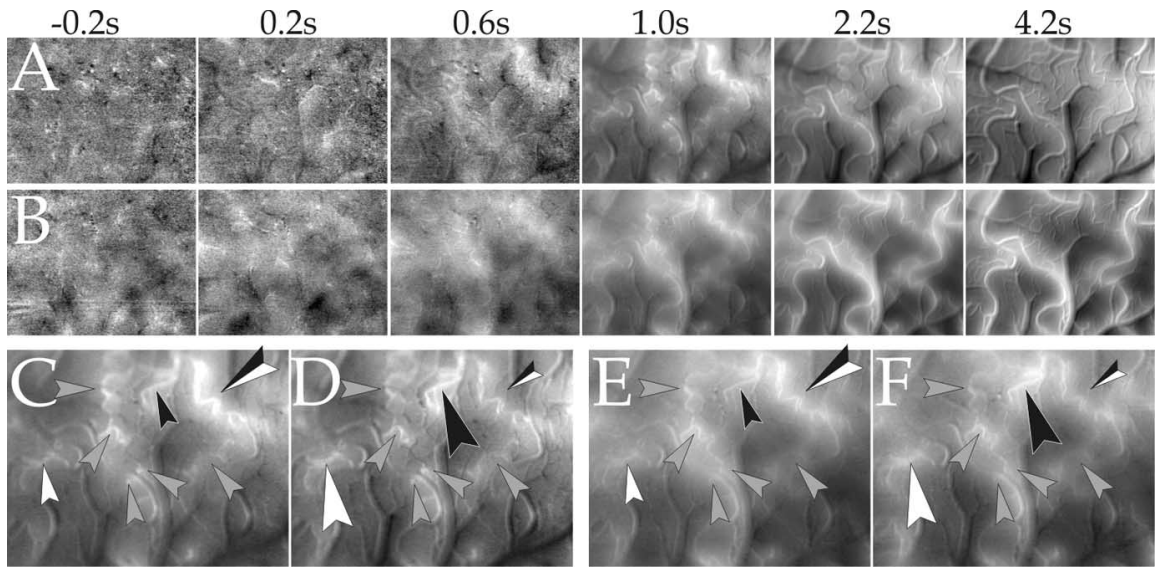

Figure 6. Full dynamic range display of the CBV response; stimulus specificity of the arteriolar activation. $\boldsymbol{A}$, Series of singlecondition maps (stimuli: horizontal gratings divided by blank) obtained during fluorescence imaging. $\boldsymbol{B}$, Same as $\boldsymbol{A}$, but reflection at $565 \mathrm{~nm}$ was used instead of fluorescence (the grayscale was inverted for ease of comparison with $\boldsymbol{A}$ ). The grayscale of each frame in $\boldsymbol{A}$ and $\boldsymbol{B}$ was centered on its mean value and stretched to $\pm 3 \mathrm{SD}$ over all pixels in the image. Clipping ranges: $0.04,0.07$, $0.1,0.5,2.7,2.6 \%(\boldsymbol{A})$ and $0.1,0.25,0.46,1.6,6.0,5.2 \%(\boldsymbol{B})$. Note that if any patterns are seen before arterioles become visible, they are unrelated between $\boldsymbol{A}$ and $\boldsymbol{B}$ (compare, for example, the second frame), thus suggesting vascular noise as their origin. $\boldsymbol{C}$, $\boldsymbol{D}$, Fluorescence response to horizontal and vertical gratings, respectively, averaged from 0.96 to $1.36 \mathrm{~s}$ after stimulus onset. $\boldsymbol{E}, \boldsymbol{F}$, Same as $\boldsymbol{C}$ and $\boldsymbol{D}$, but reflection instead of fluorescence (color map was inverted). All arrowheads mark arterioles. Note the difference in activation (emphasized by the size of the arrowheads) of the arterioles marked by the black, white, and black-andwhite arrowheads, depending on whether a horizontal or a vertical stimulus was presented ( $C$ vs $\boldsymbol{D}$ and $\boldsymbol{E}$ vs $\boldsymbol{F}$ ). Clipping range (C-F): $0.50,0.44,1.63,1.48 \%$.

that no consistent activity pattern was detected before arterioles emerged above the noise level of the measurements (Fig. 6A,B). Arteries became strongly activated after $\sim 2 \mathrm{~s}$. On the other hand, veins, venules, and the capillaries (parenchyma) were characterized by weaker signals, showing that the active arterial vasodilatation was larger than the passive inflating of capillaries, venules, and veins, despite their high compliance. Different stimuli consistently activated different arterioles to a different extent (e.g., the same differences between $C$ and $D$ can be seen between $E$ and $F$ in Fig. 6); however, as opposed to results obtained in auditory (Harel et al., 2000) and barrel cortex (Sheth et al., 2004) but in agreement with previous results obtained in the visual cortex of the anesthetized cat and the awake and anesthetized monkey (Frostig et al., 1990; Vanzetta and Grinvald, 2001; Vanzetta et al., 2002), in our study no functional patches were seen in single-condition CBV maps, at least not without further image processing (Sheth et al., 2004; Vanzetta et al., 2004).
Oximetric functional maps estimated by reflection measurements at $605 \mathrm{~nm}$

Numerous previous experiments performed in our laboratory showed that the presence or lack of "single condition" (stimulus vs blank) functional maps at the oximetric wavelength of $605 \mathrm{~nm}$ is a standard indicator for the quality of the preparation from the point of view of the SNR. The presence or absence of such maps was thus used as a criterion to keep or reject the experiment.

The results of the $605 \mathrm{~nm}$ measurements are shown in Figure 7. Data were acquired at $200 \mathrm{~ms}$ per frame and binned to $400 \mathrm{~ms}$ per frame for display purposes to compensate for the $\sim 3.3$ times lower SNR than at $565 \mathrm{~nm}$, resulting from a $>10$ times lower signal amplitude caused by the weaker absorption of hemoglobin at $605 \mathrm{~nm}$.

In the early $605 \mathrm{~nm}$ images, no activated macro-vessels could be observed. Rather, the first structures that became visible after stimulation were parenchyma patches corresponding to the functional architecture (marked by the crosses obtained from the differential map) (Fig. $8 \mathrm{~A}$ ), indicating that the absorption increase at $605 \mathrm{~nm}$ started at the loci of neuronal activity. At $605 \mathrm{~nm}$, Hbr absorbs approximately five times more than $\mathrm{HbO} 2$, and imaging at this wavelength thus emphasizes changes in Hbr concentration ([Hbr]). During the first second after stimulus onset, the CBV response was still small, and furthermore, no functional CBV-based singlecondition maps were obtained (Fig. 6). Thus we attribute the observed parenchyma patches mostly to a passive, highly local increase in [Hbr], which is attributable to an increase in oxygen extraction by the activated neurons or the surrounding glia rather than to effects of the active blood-volume response. After $\sim 2 \mathrm{~s}$, also at $605 \mathrm{~nm}$, blood vessels became predominant because of the large $\mathrm{CBV}$ and $\mathrm{CBF}$ response, as seen in previous experiments performed on anesthetized and awake preparations (Frostig et al., 1990; Grinvald et al., 2000; Nemoto et al., 2004). To increase the SNR in the functional single-condition maps, the first $0.8 \mathrm{~s}$ of

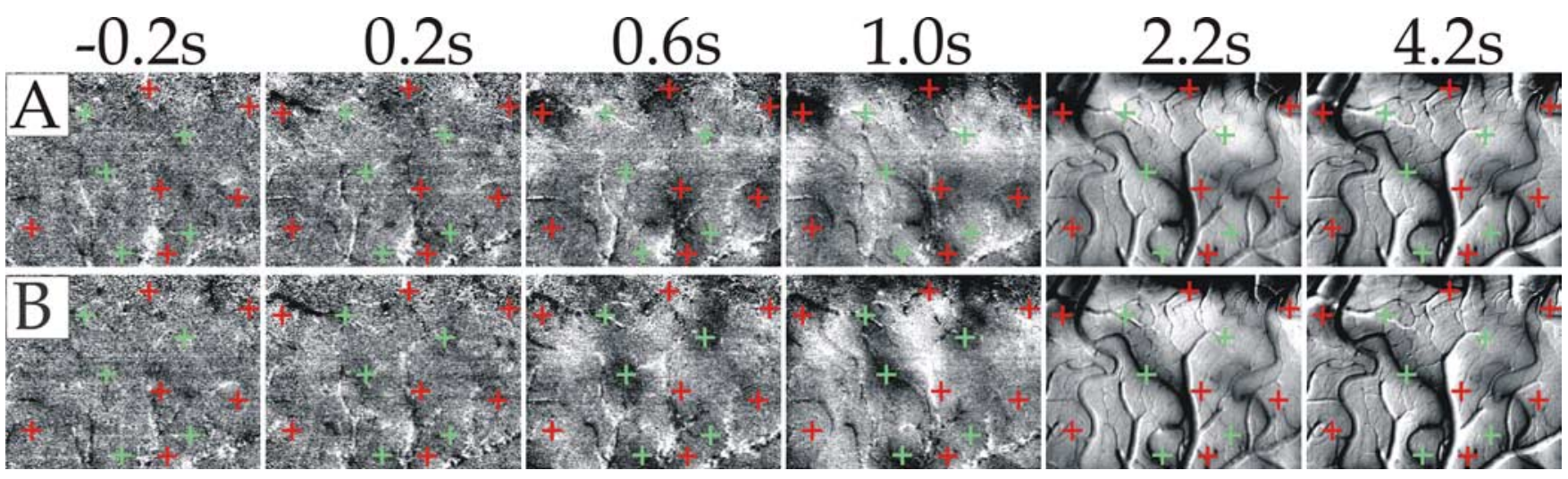

Figure 7. Single-condition maps, $605 \mathrm{~nm}$ reflection; first $4.5 \mathrm{~s}$ of the response. $\boldsymbol{A}$, Series of single-condition (vertical vs blank) reflection images. $\boldsymbol{B}$, Series of the "orthogonal" single-condition maps (horizontal vs blank). The grayscale of the maps in $\boldsymbol{A}$ and $\boldsymbol{B}$ was centered on the mean value of each image as in Figure 6 and stretched to \pm 1.5 SD. The baseline pattern (the average image from -0.8 s until stimulus onset) was subtracted from all images. In all images, the red and green crosses correspond to the location of the black and white functional patches, as obtained from the differential map in Figure 8 . 
the response were averaged and redisplayed in Figure 8, $B$ and $C$, to facilitate the comparison with the differential map (Fig. $8 A)$.

\section{Activity-dependent oximetric changes in different vascular compartments} To evaluate the differences in the [Hbr] increase in the various microvascular compartments, in Figure 8, $E$ and $F$, we show the compartment-resolved reflection time courses obtained at $605 \mathrm{~nm}$ (same display as used for Fig. 5). We zoomed into the early part of the response, because during the first second after stimulus onset the $\mathrm{CBV}$ and $\mathrm{CBF}$ responses were still small, and thus at least in capillaries, venules, and veins [Hbr] increased mostly without active intervention of the vasculature. Indeed, at $1 \mathrm{~s}$, the $605 \mathrm{~nm}$ time course (Fig. $8 D$ ) was already at $\sim 30 \%$ of its minimum, whereas the CBV signal had barely reached $10 \%$ of its peak (Fig. 4 ). In the experiments characterized by the lowest vascular noise, it was apparent that the parenchymal signal significantly preceded the other compartments in the earliest part of the time course (Figs. $8 E$, 9A, top), despite the large amplitude of the active arteriolar response. Once more, this indicates the presence of a passive [Hbr] increase in capillaries attributable to increased oxygen extraction. Because of the large amplitude of the arterial and arteriolar CBV responses (Fig. $5 A, B$ ), in the nonnormalized display, those signals increased as fast as the parenchymal one (Fig. $8 E$ ) and dominated after $\sim 1 \mathrm{~s}$. When the traces were normalized correcting for the different amplitudes at peak, however, the parenchymal/venular [Hbr] increase clearly had a faster dynamics than that taking place in the arteries and arterioles (Figs. $8 F, 9 A$, bottom). The venular signal was found to tightly follow the capillary one, as expected from the fast transfer of capillary blood into venules.

In Figures 4, 5, and 8, we showed that the intercompartmental differences in time course were statistically significant with respect to the variability of the responses among different vascular elements belonging to the same vessel type. Figure 9 shows that those intercompartmental differences were highly significant also relative to the variability that exists among trials, grouped into independent datasets (see Materials and Methods, Data analysis).

The obtained dynamics suggest that after stimulation, [Hbr] indeed increased the fastest in the capillaries. Furthermore, in the experiments characterized by the lowest vascular noise, the capillary $605 \mathrm{~nm}$ time course was found to diverge (Fig. 9A, top panel) $\sim 200 \mathrm{~ms}$ before significant differences developed among the time courses of the other compartment. These latter differlines, veins.
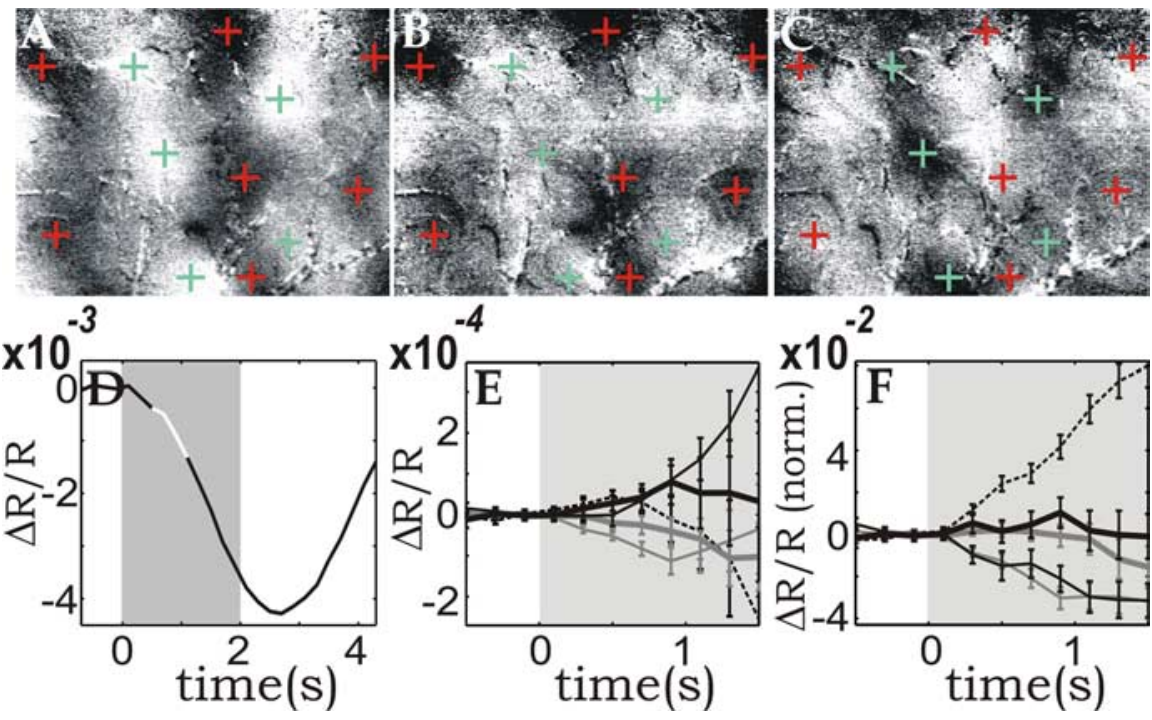

Figure 8. Averaged functional maps; compartment-resolved oximetric changes. $\boldsymbol{A}$, Differential map (vertical stimulus divided by horizontal one) averaged from 0.4 to $1.2 \mathrm{~s}$ after stimulus onset (highlighted white segment in $\boldsymbol{D}$ ). Clipping range: $0.07 \%$. Red and green crosses mark dark and bright patches to facilitate comparison between the single condition and the differential maps. $\boldsymbol{B}, \boldsymbol{C}$, Single-condition maps obtained by dividing the images obtained while a vertical $(\boldsymbol{B})$ or horizontal $(\boldsymbol{C})$ stimulus was presented by those obtained during a blank stimulus and averaged over the same time as in $\boldsymbol{A}$. Clipping range: $0.05 \%$. D, Initial decrease in the "global signal" time course (the initial dip), obtained by averaging the response over the entire image. The well known subsequent large overshoot resulting from the hyperoxygenation induced by the increased CBF is not shown here. $\boldsymbol{E}, \boldsymbol{F}$, Early time courses of reflected light at $605 \mathrm{~nm}$, resolved for microvascular compartments. As in Figure 5, the mean time course (average ove all compartments) was subtracted before display. $\boldsymbol{E}$, Non-normalized display. $\boldsymbol{F}$, After normalization (norm.) (same procedure as in Fig. 5). Dashed black lines, Arteries; thick gray lines, arterioles; thin gray lines, parenchyma; thin black lines, venules; thick black
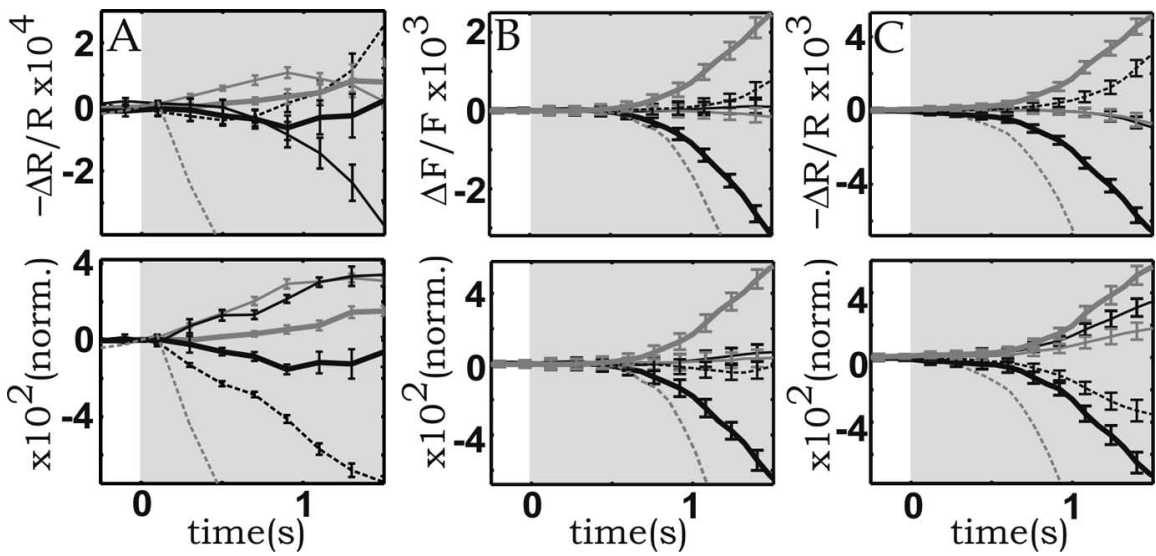

Figure 9. Reproducibility of time courses with respect to the variability over different datasets. $\boldsymbol{A}$, Reflection (R) signals at 605 $\mathrm{nm}$. $\boldsymbol{B}$, Fluorescence intensity (F). C, Reflection at $570 \mathrm{~nm}$. To facilitate timing comparisons, the bottom panels display the graphs after normalizing (norm.) the peak amplitudes to $[0,1]$. Dashed black lines, Arteries; thick gray lines, arterioles; thin gray lines, parenchyma; thin black lines, venules; thick black lines, veins. The dashed gray line in all panels depicts the true "zero signal level," which was omitted in Figures 5 and 8 for clarity of display. Note that because of the convention used in this display, relative to the zero-ordinate line, the dashed gray trace corresponds to the sign-inverted mean response (average over all compartments). 


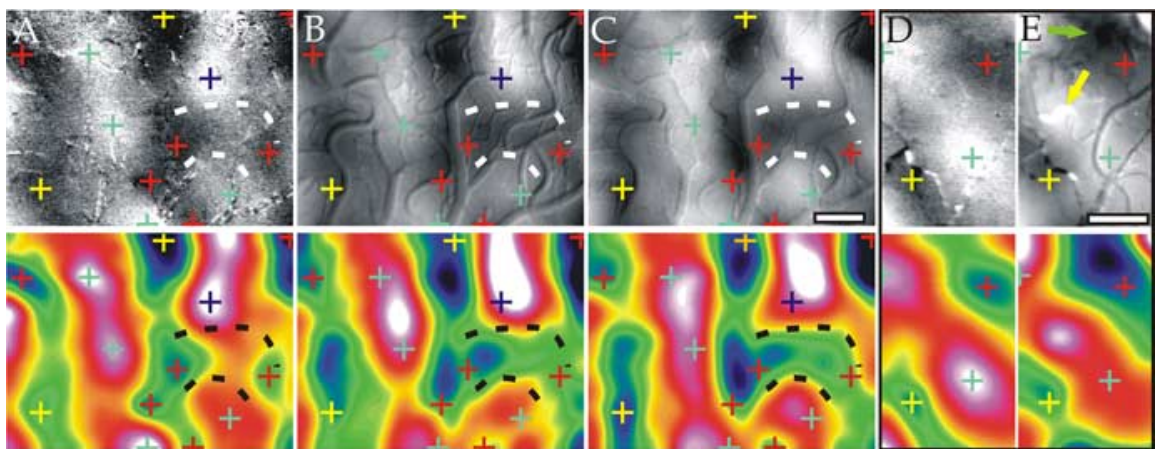

Figure 10. Comparison of differential maps recorded with different modalities. Top panels: $\boldsymbol{A}$, Differential map (vertical/ horizontal) obtained at $605 \mathrm{~nm}$. Clipping range: $0.08 \%$. $\boldsymbol{B}$, Differential map obtained with fluorescence imaging (color map in $\boldsymbol{B}$ was inverted to facilitate comparison with the other panels). Clipping range: $0.31 \%$. C, Differential map obtained at $565 \mathrm{~nm}$. Clipping range: $0.7 \%$. Although the image in $\boldsymbol{A}$ was averaged only from 0.4 to $1.2 \mathrm{~s}, \boldsymbol{B}$ and $\boldsymbol{C}$ were averaged for the duration of the CBV response $(\sim 8 \mathrm{~s})$. $\boldsymbol{D}, \boldsymbol{E}$, Differential maps (vertical/horizontal) obtained at 605 and $570 \mathrm{~nm}$ from another cat. Clipping range: 0.07 and $0.27 \%$, respectively. Arrows in $\boldsymbol{E}$ indicate two examples of stimulus-specific activated arterioles. Bottom panels: Same as top panels, but Gaussian $(\sigma \sim 100 \mu \mathrm{m})$ low-pass filtered to enhance the functional patches over the vascular patterns. As shown by the pseudocolor display, the crosses marking dark and bright patches were deduced from maxima and minima of the oximetric maps $(\boldsymbol{A}, \boldsymbol{D}$, bottom) and transposed to the identical locations in the other panels to facilitate comparison between the maps. Scale bars, $0.5 \mathrm{~mm}$.

capillary signals were always the fastest ones after the amplitudes were normalized (Fig. 9A, bottom panel), which is consistent with a fast deoxygenation taking place in those compartments.

Direct oxygen tension measurements (Thompson et al., 2003) have shown that the early parenchymal hypo-oxygenation after increased spiking activity is mostly confined to the activated cortical columns. The early [Hbr] increase occurring in the capillaries, in which the exchange with the surrounding tissue is strongest, is thus expected to be highly localized to the active columns, more than that occurring in noncapillary vessels such as venules and veins, which drain from both active and nonactive columns. Consistently, when the vessel samples for the different compartments were collected from the entire imaged area, the amplitude for the obtained capillary signal was somewhat smaller than when the samples were restricted to the active columns; furthermore, when time courses were amplitude normalized, the dynamics of the capillary signal tended to be slightly slower than the venular one (see supplemental Fig. 1, available at www.jneurosci.org as supplemental material).

\section{Comparison of CBV- and oximetry-based functional maps}

As shown previously by Frostig et al. (1990), high-resolution CBV-based functional maps could be obtained (Fig. $10 B, C$, top) when the large-amplitude, stimulus-unspecific components dominating in single-condition maps (stimulus divided by blank) (Figs. 3, 6) were removed from the signal by differential imaging (a stimulated condition divided by the "orthogonal" one: here, vertically vs horizontally orientated gratings). This shows that the CBV signal also had a capillary component that colocalized with the electrical activity. The obtained CBV maps were similar to those obtained at $605 \mathrm{~nm}$ during the initial phase of the response $(0.4-1.2 \mathrm{~s})$ (Fig. 10A, top) that have been confirmed to correlate with single-unit and multiunit spiking activity in numerous studies combining optical imaging and electrical recordings (Bonhoeffer and Grinvald, 1991; Shmuel and Grinvald, 1996; Wang et al., 1996; Weliky et al., 1996; Bosking et al., 1997); however, morphological differences in the obtained functional architecture were also observed. In some cases, these differences were clearly of capillary origin (e.g., the parenchyma patch outlined by dashed lines is absent in Fig. $10 \mathrm{~A}$ but is clearly present in $B$ and $C$ ). To quantify these differences, we calculated the correlation coefficients $(r)$ between the maps, after Gaussian low-pass filtering $(\sigma \sim 100 \mu \mathrm{m})$ (Fig. 10, bottom panels) to avoid correlation artifacts resulting from vascular activation patterns (Vanzetta et al., 2004). Although correlating CBV maps obtained during fluorescence and $565 \mathrm{~nm}$ reflection yielded $r=0.92$, correlating either of those with the map obtained during reflection at $605 \mathrm{~nm}$ yielded a lower $r=0.84$ (as a comparison, correlating a map with white noise filtered in the same way yields values of $r \sim 0.08)$. The same ordering of $r$ values was also found when the CBV maps were calculated using only the initial part of the response, for Gaussian filtering with $\sigma$ values from 90 up to $300 \mu \mathrm{m}$ (see supplemental Fig. 2, available at www.jneurosci.org as supplemental material).

Similar differences between functional patterns obtained during differential imaging using the early oximetric signal (maps obtained at $605 \mathrm{~nm}$ during the first $\sim 2 \mathrm{~s}$ ) and the active vascular response (maps obtained from the CBV signal, as well as late oximetric maps) have been found in numerous other experiments performed on the visual cortex of anesthetized cats and awake monkeys in our laboratory (Vanzetta et al., 2004) as well as in rodent barrel cortex (Erinjeri and Woolsey, 2002). Figure 10, D and $E$, show another example of an early oximetric and a CBV-based differential orientation map, respectively $(r=0.80)$ taken from another cat. Here as well, small but consistent differences in the location of the centers of the functional patches could be seen. Some morphological differences were clearly of parenchymal origin (e.g., the shape of the dark patch marked by the yellow cross) (Fig. 10D,E).

Clear stimulus-specific activation of arterioles could also be seen in all CBV maps. Two examples are marked by arrows in Figure $10 \mathrm{E}$. Although these arterioles were indeed within the "correct" rather than the "orthogonal" domain of orientation preference (Fig. $10 \mathrm{E}$, yellow arrow, horizontal; green arrow, vertical), they were displaced with respect to the locations where the preference for the horizontal or vertical orientation, respectively, was maximal as determined from the oximetric map (Fig. 10D, green and red crosses, respectively). These results demonstrate how the precise spatial estimation of the functional architecture obtained by CBV measurements is limited by the geometric properties of the underlying vascular network.

\section{Discussion}

\section{Blood-volume changes and neurovascular coupling}

Here we selectively determined the dynamics of the CBV response for the various microvascular compartments using standard visual stimulation rather than hypercapnia (Lee et al., 2001). Use of high-resolution optical imaging $(80 \mathrm{~ms}, \sim 7 \mu \mathrm{m})$ at low magnification rather than high-magnification videomicroscopybased vessel-diameter measurements enabled us to study hemodynamic responses down to the level of arterioles and venules and, by imaging the parenchyma, capillaries, and to relate them to the local functional architecture (requiring a large field of view: $\sim 2 \times 3 \mathrm{~mm}$ ).

We found that the active vascular response started at arteriolar level, in agreement with observations in rat by Nemoto et al. 
(2004). Here we scrutinized this finding more systematically. Arterioles and arteries, endowed with active regulatory mechanisms, had the largest response. We also detected a capillary CBV increase. It preceded the venous response, which exhibited the lowest amplitude. These results are inconsistent with the balloon model (Buxton and Frank, 1997; Buxton et al., 1998) because it assumes that the main blood-volume changes occur in veins, inflating passively as a result of their high compliance.

We also found that arterioles displayed a certain degree of stimulus specificity, correlating with the functional domains, in agreement with the high resolution obtained with arterial spinlabeling fMRI techniques (Duong et al., 2001).

Several mechanisms have been suggested to link the neuronal response to the active vascular dilatation, such as the release of nitric oxide (NO) from $S$-NO-hemoglobin induced by the allosteric transition from $\mathrm{HbO} 2$ to $\mathrm{Hbr}$ (Stamler et al. 1997) or the release of ATP from red RBCs at low $\mathrm{PO}_{2}$ and $\mathrm{pH}$ at the capillary/ venular level (Collins et al., 1998; Dietrich et al., 2000); however, our results indicate that the active vascular response begins at the arteriolar level. The above hypothesis would thus require an arteriolar deoxygenation or a signal spreading from capillaries/ venules toward arterioles. Although such backward communication across the capillary bed has been detected in muscle (Collins et al., 1998), in the cortex such a hypothesis is still awaiting experimental confirmation.

Alternatively, our results are also consistent with a more direct link between neuronal activity and the active vascular response, for example, that mediated by the vasodilatatory effect of $\mathrm{K}^{+}$ ions, together with modulatory effects of $\mathrm{H}^{+}$and adenosine (Kuschinsky, 2000). Anatomic studies have shown the existence of pre-arteriolar sphincters (pericytes wrapped around the vessels), raising the possibility of a link between spiking and vascular response by direct innervation of those cells (Harrison et al. 2002). Astrocytes have also been proposed to be involved in the link between neuronal metabolism and oxygen/glucose supply by the blood (Magistretti et al., 1994; Pellerin and Magistretti, 1994, 2004; Magistretti and Pellerin, 1999; Shulman et al., 2001), although the involved time constants (seconds) are somewhat slow (but see Zonta et al., 2003). The fast dynamics revealed here provide some constraints on the proposed hypotheses.

\section{Oximetric changes}

Using oxygen-electrode measurements, Ances et al. (2001) detected a stimulus-evoked decrease in tissue oxygen tension, tightly colocalized with spiking activity (Thompson et al., 2003). An activity-induced $\mathrm{PO}_{2}$ decrease has also been directly detected in the cortical microvasculature (Vanzetta and Grinvald, 1999). Because in the cat cortex it precedes the active vascular response by $\sim 300 \mathrm{~ms}$, we interpreted this blood deoxygenation as resulting from increased capillary oxygen extraction (Vanzetta and Grinvald, 2001) rather than from the effects of CBV or CBF alone (Buxton et al., 1998), and indeed a recent extension of the balloon model includes this scenario (Buxton et al., 2004). The present compartment-resolved $605 \mathrm{~nm}$ data support this interpretation. Indeed, the early $605 \mathrm{~nm}$ signal decreased faster in capillaries than in the other compartments (Fig. 9). Furthermore, it was the fastest optical signal detected.

\section{Activity-dependent local changes in hematocrit}

Even after correcting the fluorescence for the effects of absorption changes at excitation wavelength, the obtained CBV time course was slower than the $570 \mathrm{~nm}$ reflection signal. A possible explanation for this delay is a sensory-evoked RBC density de- crease, as observed by Kleinfeld et al. (1998) in single cortical vessels of the rat (but see Chaigneau et al., 2003). The somewhat slower dynamics of hematocrit changes suggested by our measurements might result from the larger average cortical capillary length in the cat. The difference in penetration depth of green versus red light might also have contributed to the differences between the two time courses, in particular because the two signals were averaged over the entire imaged area rather than taken from individual vessels. Additional experiments are required to assess the relative amplitude of those effects as well as that of oximetric changes at fluorescence emission wavelength and eventually of a small oximetric dependence of the $570 \mathrm{~nm}$ reflection signal.

\section{Implications for functional brain imaging}

From the mapping point of view, passive oxygen extraction attributable to increased neuronal metabolism obviously correlates highly with electrical activity, whereas the active vascular reaction imposes complex spatial filtering on the neuronal activity patterns, depending on the properties and geometry of the local vascular network, especially its noncapillary part. Indeed, functional modules are columnar structures extending from the pial surface to the white matter, embedded in the bulk of the gray matter just like the capillary network, which is therein distributed approximately isotropically with an intervessel distance of $\sim 25$ $\mu \mathrm{m}$ (Pawlik et al., 1981). Most capillaries thus remain within the same cortical module (median capillary length, $\sim 110 \mu \mathrm{m}$; median curvature radius, $\sim 60 \mu \mathrm{m}$ ) (Pawlik et al., 1981). On the other hand, noncapillary vessels are not only much sparser, but also run preferentially in parallel with the cortical sheet, thus often crossing the boundaries of the columnar domains. The resulting limitations on the spatial resolution that are achievable are particularly relevant in the case of multiple columnar systems coding for different functional features and coexisting in a square millimeter of cortex. Examples are the spatially overlapping orientation, ocular dominance, and cytochrome oxidase blobs systems in primate V1, characterized by topologically unconnected activity patterns, as opposed to cortical areas organized according to either a single columnar system, such as rodent barrel cortex in which the vascular layout correlates with the functional architecture (Cox et al., 1993), or to areas organized according to topologically connected activity patches, the cortical localization of which shifts gradually with varying stimulus characteristics as in auditory cortex (Harel et al., 2000; Versnel et al., 2002). Thus, in the general case, because of the dominant noncapillary component, there are anatomical limits to the resolution of the CBV and CBF signals, unless their capillary component is enhanced. This is possible, for instance, by using high-field Hahn spin echo blood oxygenation level-dependent (BOLD) fMRI (Yacoub et al., 2003) or by crushing the noncapillary contribution of the fMRI signal using extrinsic contrast agent-based fMRI (Harel et al., 2002). In fact, $\mathrm{CBV}$ changes contain a local component, as first shown by high-resolution differential maps of the functional architecture obtained at isosbestic wavelengths with optical imaging (Frostig et al., 1990) and in differential positive-BOLD fMRI studies (Menon et al., 1997; Cheng et al., 2001; Goodyear and Menon, 2001).

Our results also show directly that arterioles have stimulusspecific responses. Despite their lower spatial density as compared with that of capillaries, the elevated arteriolar flow changes make this compartment interesting for somewhat lower resolution flow-based fMRI relative to that based on signals of capillaries. Indeed, using the flow-sensitive alternating inversion recovery technique, Duong et al. (2001) obtained functional maps at a 
spatial resolution comparable with that of imaging techniques using the "initial dip" BOLD signal (Hu et al., 1997; Kim et al. 2000), which, although tightly linked to neuronal activity, suffers from poor SNR because of the small amplitude of the initial dip BOLD-fMRI signal and the short time available for data acquisition (Duong et al., 2000; Ugurbil et al., 2003).

Finally, we found small but consistent morphological discrepancies between CBV-based and hypo-oxygenation-based functional maps. To a certain extent, those discrepancies result from a different geometry of the vascular compartments dominating each imaged hemodynamic signal (arterioles vs capillaries); however, geometric considerations are unlikely to explain the discrepancies observed at the level of parenchyma patches. Recent evidence suggests that the active vascular response (underlying the positive BOLD signal) reflects synaptic rather than spiking activity (Caesar et al., 2003a,b; Logothetis, 2003), whereas the stimulus-evoked tissue deoxygenation underlying the initial dip has been found to correlate, at least spatially, with spiking activity (Thompson et al., 2003). Furthermore, Sheth et al. (2003) and Nemoto et al. (2004) found that field potential measurements correlated differently with the $570 \mathrm{~nm}$ and the early $605 \mathrm{~nm}$ optical signals. This raises the question of whether some of the observed differences between the functional maps obtained using the initial hypo-oxygenation and those obtained using the CBV response might not result from a different coupling of the two hemodynamic processes to spiking and synaptic activity, respectively. During full-field stimulation, spiking is spatially correlated with the local presynaptic and postsynaptic currents; the detected differences are thus expected to be small, which is consistent with our data. This underscores the importance of developing experimental protocols in which spiking and subthreshold activity patterns that are a priori known to be different are selectively influenced, for example, by using pharmacological manipulations (Caesar et al., 2003b) or retinotopic stimuli activating a small portion of the imaged area.

\section{References}

Ances BM, Buerk DG, Greenberg JH, Detre JA (2001) Temporal dynamics of the partial pressure of brain tissue oxygen during functional forepaw stimulation in rats. Neurosci Lett 306:106-110.

Bonhoeffer T, Grinvald A (1991) Iso-orientation domains in cat visual cortex are arranged in pinwheel-like patterns. Nature 353:429-431.

Bonhoeffer T, Grinvald A (1993) The layout of iso-orientation domains in area 18 of cat visual cortex: optical imaging reveals a pinwheel-like organization. J Neurosci 13:4157-4180.

Bosking WH, Zhang Y, Schofield B, Fitzpatrick D (1997) Orientation selectivity and the arrangement of horizontal connections in tree shrew striate cortex. J Neurosci 17:2112-2127.

Buxton RB, Frank LR (1997) A model for the coupling between cerebral blood flow and oxygen metabolism during neural stimulation. J Cereb Blood Flow Metab 17:64-72.

Buxton RB, Wong EC, Frank LR (1998) Dynamics of blood flow and oxygenation changes during brain activation: the balloon model. Magn Reson Med 39:855-864.

Buxton RB, Uludag K, Dubowitz DJ, Liu TT (2004) Modeling the hemodynamic response to brain activation. NeuroImage 23:S220-233.

Caesar K, Gold L, Lauritzen M (2003a) Context sensitivity of activitydependent increases in cerebral blood flow. Proc Natl Acad Sci USA 100:4239-4244.

Caesar K, Thomsen K, Lauritzen M (2003b) Dissociation of spikes, synaptic activity, and activity-dependent increments in rat cerebellar blood flow by tonic synaptic inhibition. Proc Natl Acad Sci USA 100:16000-16005.

Chaigneau E, Oheim M, Audinat E, Charpak S (2003) Two-photon imaging of capillary blood flow in olfactory bulb glomeruli. Proc Natl Acad Sci USA 100:13081-13086.

Chatton JY, Pellerin L, Magistretti PJ (2003) GABA uptake into astrocytes is not associated with significant metabolic cost: implications for brain im- aging of inhibitory transmission. Proc Natl Acad Sci USA 100:12456-12461.

Cheng K, Waggoner RA, Tanaka K (2001) Human ocular dominance columns as revealed by high-field functional magnetic resonance imaging. Neuron 32:359-374.

Collins DM, McCullough WT, Ellsworth ML (1998) Conducted vascular responses: communication across the capillary bed. Microvasc Res 56:43-53.

Cox SB, Woolsey TA, Rovainen CM (1993) Localized dynamic changes in cortical blood flow with whisker stimulation corresponds to matched vascular and neuronal architecture of rat barrels. J Cereb Blood Flow Metab 13:899-913.

Dietrich HH, Ellsworth ML, Sprague RS, Dacey RGJ (2000) Red blood cell regulation of microvascular tone through adenosine triphosphate. Am J Physiol Heart Circ Physiol 278:H1294-H1298.

Duong TQ, Kim D-S, Ugurbil K, Kim S-G (2000) Spatiotemporal dynamics of the BOLD fMRI signals: toward mapping submillimeter cortical columns using the early negative response. Magn Reson Med 44:231-242.

Duong TQ, Kim D-S, Ugurbil K, Kim S-G (2001) Localized cerebral blood flow response at submillimeter columnar resolution. Proc Natl Acad Sci USA 98:10904-10909.

Erinjeri JP, Woolsey TA (2002) Spatial integration of vascular changes with neural activity in mouse cortex. J Cereb Blood Flow Metab 22:353-360.

Frostig RD, Lieke EE, Ts'o DY, Grinvald A (1990) Cortical functional architecture and local coupling between neuronal activity and the microcirculation revealed by in vivo high-resolution optical imaging of intrinsic signals. Proc Natl Acad Sci USA 87:6082-6086.

Goodyear BG, Menon RS (2001) Brief visual stimulation allows mapping of ocular dominance in visual cortex using fMRI. Hum Brain Mapp 14:210-217.

Grinvald A, Manker A, Segal M (1982) Visualization of the spread of electrical activity in rat hippocampal slices by voltage-sensitive optical probes. J Physiol (Lond) 333:269-291.

Grinvald A, Lieke E, Frostig RD, Gilbert CD, Wiesel TN (1986) Functional architecture of cortex revealed by optical imaging of intrinsic signals. Nature 324:361-364.

Grinvald A, Slovin H, Vanzetta I (2000) Non-invasive visualization of cortical columns by f-MRI. Nat Neurosci 3:105-107.

Harel N, Mori N, Sawada S, Mount RJ, Harrison RV (2000) Three distinct auditory areas of cortex (AI, AII, and AAF) defined by optical imaging of intrinsic signals. NeuroImage 11:302-312.

Harel N, Zhao F, Wang P, Kim SG (2002) Layer specificity of BOLD and CBV fMRI signals at ultra-high resolution [abstract]. Conference on Functional Mapping of the Human Brain. NeuroImage 16:1221-1223.

Harrison RV, Harel N, Panesar J, Mount RJ (2002) Blood capillary distribution correlates with hemodynamic-based functional imaging in cerebral cortex. Cereb Cortex 12:225-233.

Hill DK, Keynes RD (1949) Opacity changes in stimulated nerve. J Physiol (Lond) 108:278-281.

Hu X, Le TH, Ugurbil K (1997) Evaluation of the early response in fMRI in individual subjects using short stimulus duration. Magn Reson Med 37:877-884.

Kim DS, Doung TQ, Kim SG (2000) High-resolution mapping of isoorientation columns by fMRI. Nat Neurosci 3:164-199.

Kleinfeld D, Mitra PP, Helmchen F, Denk W (1998) Fluctuations and stimulus-induced changes in blood flow observed in individual capillaries in layers 2 through 4 of rat neocortex. Proc Natl Acad Sci USA 95:15741-15746.

Kohl M, Lindauer U, Royl G, Kühl M, Gold L, Villringer A, Dirnagl U (2000) Physical model for the spectroscopic analysis of cortical intrinsic optical signals. Phys Med Biol 45:3749-3764.

Kuschinsky W (2000) Regulation of cerebral blood Flow. In: Functional MRI (medical radiology — diagnostic imaging and radiation oncology) (Moonen CTW, Bandettini PA, eds). pp 15-25. New York: Springer.

Lee SP, Duong TQ, Yang G, Iadecola C, Kim SG (2001) Relative changes of cerebral arterial and venous blood volumes during increased cerebral blood flow: implications for BOLD fMRI. Magn Reson Med 45:791-800.

Lindauer U, Royl G, Leithner C, Kühl M, Gold L, Gethmann J, Kohl-Bareis M, Villringer A, Dirnagl U (2001) No evidence for early decrease in blood oxygenation in rat whisker cortex in response to functional activation. NeuroImage 13:988-1001. 
Lipton P (1973) Effects of membrane depolarization on light scattering by cerebral cortical slices. J Physiol (Lond) 231:365-383.

Logothetis NK (2000) Can current fMRI techniques reveal the microarchitecture of cortex? Nat Neurosci 3:413.

Logothetis NK (2003) The underpinnings of the BOLD functional magnetic resonance imaging signal. J Neurosci 23:3963-3971.

MacVicar BA, Hochman D (1991) Imaging of synaptically evoked intrinsic optical signals in hippocampal slices. J Neurosci 11:1458-1469.

Magistretti PJ, Pellerin L (1999) Astrocytes couple synaptic activity to glucose utilization in the brain. News Physiol Sci 14:177-182.

Magistretti PJ, Sorg O, Naichen Y, Pellerin L, de Rham S, Martin JL (1994) Regulation of astrocyte energy metabolism by neurotransmitters. Ren Physiol Biochem 17:168-171.

Magistretti JP, Pellerin L, Rothman DL, Shulman RG (1999) Energy on demand. Science 283:496-497.

Malonek D, Grinvald A (1996) Interactions between electrical activity and cortical microcirculation revealed by imaging spectroscopy: implications for functional brain mapping. Science 272:551-554.

Malonek D, Dirnagl U, Lindauer U, Yamada K, Kanno I, Grinvald A (1997) Vascular imprints of neuronal activity: relationships between the dynamics of cortical blood flow, oxygenation, and volume changes following sensory stimulation. Proc Natl Acad Sci USA 94:14826-14831.

Mayhew J, Zheng Y, Hou Y, Vuksanovic B, Berwick J, Askew S, Coffey P (1999) Spectroscopic analysis of changes in remitted illumination: the response to increased neural activity in brain. NeuroImage 10:304-326.

Mayhew J, Johnston D, Martindale J, Jones M, Berwick J, Zheng Y (2001) Increased oxygen consumption following activation of brain: theoretical footnotes using spectroscopic data from barrel cortex. NeuroImage 13:975-987.

Mc Loughlin NP, Blasdel GG (1998) Wavelength-dependent differences between optically determined functional maps from macaque striate cortex. NeuroImage 7:326-336.

Menon RS, Ogawa S, Strupp JP, Ugurbil K (1997) Ocular dominance in human V1 demonstrated by functional magnetic resonance imaging. J Neurophysiol 77:2780-2787.

Nemoto M, Sheth S, Guiou M, Pouratian N, Chen JW, Toga AW (2004) Functional signal- and paradigm-dependent linear relationships between synaptic activity and hemodynamic responses in rat somatosensory cortex. J Neurosci 24:3850-3861.

Obrig H, Israel H, Kohl-Bareis M, Uludag K, Wenzel R, Muller B, Arnold G, Villringer A (2002) Habituation of the visually evoked potential and its vascular response: implications for neurovascular coupling in the healthy adult. NeuroImage 17:1-18.

Orbach HS (1988) Monitoring electrical activity in rat cerebral cortex. In: Spectroscopic membrane probes, Vol 3 (Lowe LM, ed), pp 115-136. Boca Raton, FL: CRC.

Orbach HS, Cohen LB, Grinvald A (1985) Optical mapping of electrical activity in rat somatosensory and visual cortex. J Neurosci 5:1886-1895.

Parri R, Crunelli V (2003) An astrocyte bridge from synapse to blood flow. Nat Neurosci 6:5-6.

Patel U (1983) Non-random distribution of blood vessels in the posterior region of the rat somatosensory cortex. Brain Res 289:65-70.

Pawlik G, Rackl A, Bing RJ (1981) Quantitative capillary topography and blood flow in the cerebral cortex of cats: an in vivo microscopic study. Brain Res 208:35-58.

Pellerin L, Magistretti PJ (1994) Glutamate uptake into astrocytes stimulates aerobic glycolysis: a mechanism coupling neuronal activity to glucose utilization. Proc Natl Acad Sci USA 91:10625-10629.
Pellerin L, Magistretti JP (2004) Neuroenergetics: calling during astrocytes to satisfy hungry neurons. The Neuroscientist 10:53-62.

RatzlaffEH, Grinvald A (1991) A tandem-lens epifluorescence macroscope: hundred-fold brightness advantage for wide-field imaging. J Neurosci Methods 36:127-137.

Rector DM, Rogers RF, Schwaber JS, Harper RM, George JS (2001) Scattered-light imaging in vivo tracks fast and slow processes of neurophysiological activation. NeuroImage 14:977-994.

Roy C, Sherrington C (1890) On the regulation of the blood supply of the brain. J Physiol (Lond) 11:85-108.

Sheth SA, Nemoto M, Guiou M, Walker M, Pouratian N, Toga AW (2003) Evaluation of coupling between optical intrinsic signals and neuronal activity in rat somatosensory cortex. NeuroImage 19:884-894.

Sheth SA, Nemoto M, Guiou M, Walker M, Pouratian N, Hageman N, Toga AW (2004) Columnar specificity of microvascular oxygenation and volume responses: implications for functional brain mapping. J Neurosci 24:634-641.

Shmuel A, Grinvald A (1996) Functional organization for direction of motion and its relationship to orientation maps in cat area 18. J Neurosci 16:6945-6964.

Shulman RG, Hyder FL, Rothman DL (2001) Cerebral energetics and the glycogen shunt: neurochemical basis of functional imaging. Proc Natl Acad Sci USA 98:6417-6422.

Stamler JS, Jia L, Eu JP, McMahon TJ, Demchenko IT, Bonaventura J, Gernert K, Piantadosi CA (1997) Blood flow regulation by S-nitrosohemoglobin in the physiological oxygen gradient. Science 276:2034-2037.

Thompson JK, Peterson MR, Freeman RD (2003) Single-neuron activity and tissue oxygenation in the cerebral cortex. Science 299:1070-1072.

Ugurbil K, Toth L, Kim DS (2003) How accurate is magnetic resonance imaging of brain function? Trends Neurosci 26:1108-1114.

Vanzetta I, Grinvald A (1999) Increased cortical oxidative metabolism due to sensory stimulation: implications for functional brain imaging. Science 286:1555-1558.

Vanzetta I, Grinvald A (2001) Evidence and no evidence for the initial dip in the anesthetized rat; implications for human functional brain imaging. NeuroImage 13:959-967.

Vanzetta I, Slovin H, Grinvald A (2002) Spatio-temporal characteristics of neurovascular coupling in the anesthetized cat and the awake monkey. In: Brain activation and CBF control (Tomita M, Kanno I, Hamel E, eds), pp 145-155. Tokyo: Elsevier.

Vanzetta I, Slovin H, Omer DB, Grinvald A (2004) Columnar resolution of blood volume and oximetry functional maps in the behaving monkey: implications for fMRI. Neuron 42:843-854.

Versnel H, Mossop JE, Mrsic-Flogel TD, Ahmed B, Moore DR (2002) Optical imaging of intrinsic signals in ferret auditory cortex: responses to narrowband sound stimuli. J Neurophysiol 88:1545-1558.

Villringer A, Dirnagl U (1995) Coupling of brain activity and cerebral blood flow: basis of functional neuroimaging. Cerebrovasc Brain Metab Rev $7: 240-276$.

Wang G, Tanaka K, Tanifuji M (1996) Optical imaging of functional organization in the monkey inferotemporal cortex. Science 272:1665-1668.

Weliky M, Bosking WH, Fitzpatrick D (1996) A systematic map of direction preference in primary visual cortex. Nature 379:725-728.

Yacoub E, Duong TQ, Van De Moortele PF, Lindquist M, Adriany G, Kim SG, Ugurbil K, Hu X (2003) Spin-echo fMRI in humans using high spatial resolutions and high magnetic fields. Magn Reson Med 49:655-664.

Zonta M, Angulo MC, Gobbo S, Rosengarten B, Hossmann KA, Pozzan T, Carmignoto G (2003) Neuron-to-astrocyte signaling is central to the dynamic control of brain microcirculation. Nat Neurosci 6:43-50. 